\title{
A Spectrally-Accurate FVTD Technique for Complicated Amplification and Reconfigurable Filtering EMC Devices
}

\author{
Nikolaos V. Kantartzis ${ }^{1}$, Stylianos D. Assimonis ${ }^{1}$, Antonios X. Lalas ${ }^{1}$, \\ Jonathan B. Scott ${ }^{2}$, and Christos S. Antonopoulos ${ }^{1}$ \\ ${ }^{1}$ Department of Electrical and Computer Engineering, Aristotle University of Thessaloniki, GR-54124 Thessaloniki, Greece \\ ${ }^{2}$ School of Engineering, The University of Waikato, Hamilton 3240, New Zealand
}

\begin{abstract}
The consistent and computationally economical analysis of demanding amplification and filtering structures is introduced in this paper via a new spectrally-precise finite-volume time-domain algorithm. Combining a family of spatial derivative approximators with controllable accuracy in general curvilinear coordinates, the proposed method employs a fully conservative field flux formulation to derive electromagnetic quantities in areas with fine structural details. Moreover, the resulting 3-D operators assign the appropriate weight to each spatial stencil at arbitrary media interfaces, while for periodic components the domain is systematically divided to a number of nonoverlapping subdomains. Numerical results from various real-world configurations verify our technique and reveal its universality.
\end{abstract}

Index Terms_Electromagnetic compatibility, FVTD methods, microwave components, numerical schemes, reconfigurable devices.

\section{INTRODUCTION}

$\mathrm{T}$ HE development of precise time-domain algorithms for intricate electromagnetic compatibility (EMC) devices is still an open topic of the relevant scientific research. Amid several applications, amplification components and reconfigurable filters are deemed crucial in the fabrication of modern microwave systems [1]-[4]. Considering that the majority of these structures must be redesigned to comply with the constantly evolving standards, it becomes apparent that the use of consistent models can be a powerful means to decrease high production costs. Nonetheless, such a procedure is usually rather complicated, since most of the contemporary systems have fine geometries that require excessive computational resources for their simulation. To this end, different methods - opting for inherent-error enhancements - have been hitherto formulated [5]-[7]. Also, from a practical perspective, a method which can provide a viable solution for problems involving certain arrangements, like triode amplifiers, has been discussed in [8].

Based on the above issues, it is the purpose of this paper to present a 3-D finite-volume time-domain (FVTD) method with an improved spectral resolution for the accurate modeling of complex EMC devices. Two typical categories are the amplification triodes and the microstrip filters that incorporate electromagnetic bandgaps (EBGs) or networks of metamaterial resonator-wire substrates in periodic uniform or non-uniform patterns. The novel technique launches derivative approximators of adjustable order according to a conservation-law concept, which expresses the unknown quantities as components of one solution vector. Furthermore, arbitrary media interfaces can be efficiently manipulated, unlike existing approaches, by weighting all neighboring spatial stencils with specific coefficients. Concerning periodicity, the proposed scheme separates the computational space in a number of non-overlapping subdomains, where the grids retain the traditional Yee-like electric and magnetic field duality. In particular, for problems with axial symmetry, these subdomains are formed by consecutive cylin-

Manuscript received July 1, 2011. Corresponding author: C. S. Antonopoulos (e-mail: chanto@auth.gr).

Digital Object Identifier inserted by IEEE.

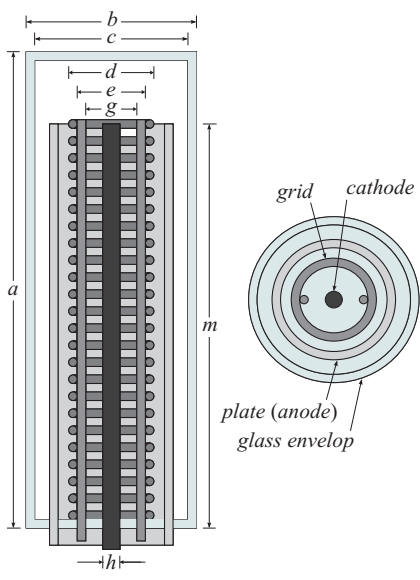

(a)

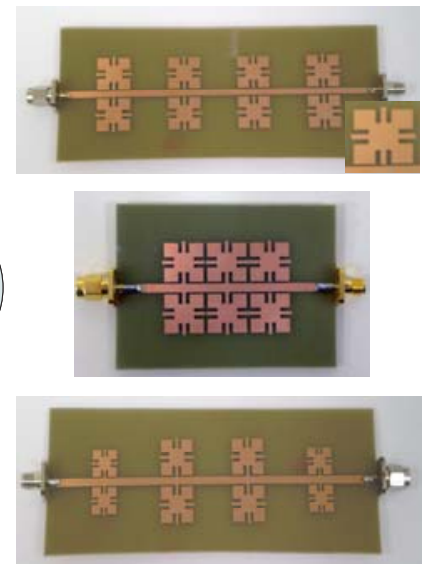

(b)
Fig. 1. (a) Geometry of a typical triode. (b) Fabricated prototypes of three uniform/non-uniform cross EBG-loaded reconfigurable filters.

drical surfaces along the main radial direction, while for planar symmetries, they usually include one unit cell. For our algorithm to be reliably certified, numerical data are selectively compared with measurements from fabricated prototypes. Results prove the stability and high precision of the method, even for hard-to-model real-world setups and large simulation times.

\section{Spectrally-Adjustable FVTD Schemes}

The proposed methodology introduces a curvilinear dual-cell mesh with higher-order derivative approximations of controllable spectral accuracy. Moreover, it employs a stable integration like that of the finite-difference time-domain (FDTD) concept. Incorporating the basic FVTD concept [9], [10], the novel algorithm involves cells with the four corners of their faces not necessarily lying on the same plane. To this goal, it is important to carefully determine all edges and faces in the resulting primary and dual grid. Hence, the suitable unit vector direction cosines are utilized to transform Maxwell's equations in the form of

$$
\varepsilon \frac{\partial \mathbf{E}}{\partial t}-\mathcal{W}[\mathbf{H}]+\sigma \mathbf{E}=-\mathbf{J}_{s} \quad \text { and } \quad \mu \frac{\partial \mathbf{H}}{\partial t}+\mathcal{W}[\mathbf{E}]=\mathbf{0},
$$

which can be deemed equivalent to the main conservation-law formulation. In (1) and assuming a general coordinate system 
$(u, v, w)$ at a homogeneous, isotropic, and lossy medium, $\mathbf{E}$ and $\mathbf{H}$ represent the electric and magnetic field intensity, respectively, while $\mathbf{J}_{s}$ is the source vector of all external excitations. Moreover, the parametric operator $\mathcal{W}[$.] is denoted as

$$
\mathcal{W}[\mathbf{F}]=\left[\begin{array}{c}
S_{v}^{L} F_{w}-S_{w}^{L} F_{v} \\
S_{w}^{L} F_{u}-S_{u}^{L} F_{w} \\
S_{u}^{L} F_{v}-S_{v}^{L} F_{u}
\end{array}\right]+\sum_{i=1}^{L}\left(q_{u} S_{u}^{L}+q_{v} S_{v}^{L}+q_{w} S_{w}^{L}\right) \mathbf{F},
$$

in which $\mathbf{F}=[\mathbf{E}, \mathbf{H}]^{T}$ with the second term launching the new $L$ th-order approximation forms that correct existing stencils around dissimilar media interfaces or slight geometric details, leading to optimal discretizations. Parameters $q_{\eta}$ with $\eta \in(u, v, w)$ guarantee the stability of the schemes, mitigate potential latetime exponential oscillations [11], and are determined by

$$
q_{\eta}=(2 L+1)(\Delta \eta)^{L}-0.5 L(\Delta \eta)^{L-1},
$$

where $\Delta \eta \in(\Delta u, \Delta v, \Delta w)$ is the proper spatial increment. To this end, spatial derivatives in (2) are computed via enhanced operators, whose spectral resolution is of order $L$. Thus, we define

$$
S_{\eta}^{L}\left[\left.F\right|_{u, v, w} ^{t}\right]=\frac{e^{L \Delta \eta / 6}}{2 \sqrt{\pi} \Delta \eta} M_{\eta, P}^{L}\left[\left.F\right|_{u, v, w} ^{t}\right]+\left.\sum_{\kappa=1}^{3}\left(1+\frac{\Delta \eta}{4 \kappa}\right) F\right|_{\eta \pm \kappa \Delta \eta} ^{t},
$$

Also, spatial form $M_{\eta, P}[$.$] enables the fine adjustment of spec-$ tral accuracy via diverse stencils and smoothness summation limit $P$. Specifically, considering the $g(u, v, w)$ system metrics,

$$
M_{\eta, P}^{L}\left[\left.F\right|_{u, v, w} ^{t}\right]=g(u, v, w) \sum_{l=1}^{L} \Lambda_{l}^{\eta}\left\{\left.\sum_{p=1}^{P} N_{l, p}^{\eta} F\right|_{u, v, w} ^{t}\right\} .
$$

Structural parameters $\Lambda_{l}$ and $N_{l, p}$ increase the consistency of the algorithm at complex geometric details or material discontinuities, like in the realistic EMC devices of Fig. 1. They are promptly determined through the subsequent expressions

$$
\Lambda_{l}^{\eta}=e^{L / 2} \sinh \left[\sqrt{1-(l \Delta \eta / 2)^{2}}\right], \quad N_{l, p}^{\eta}=(l \Delta \eta)^{P} \sqrt{2 p^{2}-1},
$$

which provide rapid interface extrapolations with all possible topologies. Having described the primary ideas of spatial derivative calculation, Maxwell's equations can be written as

$$
\partial_{t} \mathbf{F}+\mathbf{b}^{-1} \mathbf{Q F}=\mathbf{b}^{-1}(\mathbf{L}+\mathbf{R F}),
$$

$$
\text { for } \quad \mathbf{Q F}=\left[\begin{array}{c}
-\nabla \times \mathbf{H} \\
\nabla \times \mathbf{E}
\end{array}\right], \quad \mathbf{L}=\left[\begin{array}{c}
-\mathbf{J}_{s} \\
\mathbf{0}
\end{array}\right], \quad \mathbf{R}=\left[\begin{array}{cc}
-\sigma \mathbf{I} & \mathbf{0} \\
\mathbf{0} & \mathbf{0}
\end{array}\right],
$$

$\mathbf{b}=\operatorname{diag}\{\varepsilon \mathbf{I}, \mu \mathbf{I}\}$, and $\mathbf{I}$ the identity matrix. If the computational domain $\Omega$ is divided into a preselected number of cells $\Omega_{i}$ with a boundary of $\partial \Omega_{i}$, then (7) can be successfully integrated over each $\Omega_{i}$ and, after some calculus, yields

$$
\begin{aligned}
\iiint_{V_{i}} \partial_{t} \mathbf{F} d V & +\iint_{\partial \Omega_{i}} \mathbf{b}^{-1} \mathbf{\Xi}\left[\mathbf{n}_{0}\right] \mathbf{F} d \mathbf{s} \\
& =\iiint_{V_{i}} \mathbf{b}^{-1} \mathbf{L} d \mathbf{r}+\iiint_{V_{i}} \mathbf{b}^{-1} \mathbf{R} \mathbf{F} d \mathbf{r}, \\
\Xi\left[\mathbf{n}_{0}\right] & =\left[\begin{array}{cc}
\mathbf{0} & -\mathcal{W}\left[\mathbf{n}_{0}\right] \\
\mathcal{W}\left[\mathbf{n}_{0}\right] & \mathbf{0}
\end{array}\right],
\end{aligned}
$$

with

$V_{i}$ the volume of $\Omega_{i}$, and $\mathbf{n}_{0}$ the outward unit vector perpendicular to surface $\partial \Omega_{i}$. Notice that quantity $\boldsymbol{\Xi}\left[\mathbf{n}_{0}\right] \mathbf{F}$ is actually the flux through surface $\partial \Omega_{i}$. To exploit all fluxes through the cell faces, the concept of the cell average [10], [12] - i.e. the avera-
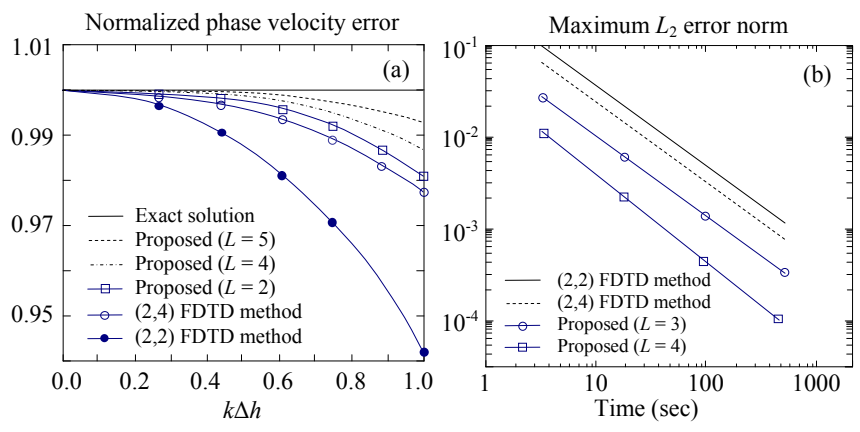

Fig. 2. (a) Normalized phase velocity error and (b) maximum $L_{2}$ error norm for the proposed and various higher-order time-domain realizations.

ge of $\mathbf{F}$ located at the barycenter of every cell - is denoted as

$$
F_{i}=V_{i}^{-1} \iiint_{V_{i}} \mathbf{F}(\mathbf{r}) d V
$$

where $\mathbf{r}$ is the position vector. For the required flux splitting, matrix $\mathbf{T}\left[\mathbf{n}_{0}\right]=\mathbf{b}^{-1} \boldsymbol{\Xi}\left[\mathbf{n}_{0}\right]$ (decomposable to a sum of matrices with positive and negative eigenvalues) is also utilized. So, and bearing in mind that the eigenvalues of $\mathbf{T}\left[\mathbf{n}_{0}\right]$ are given by $\operatorname{diag}\{0,0, v, v,-v,-v\}$ for $v=(\mu \varepsilon)^{-1 / 2}$ and $\mathcal{K}=\mathcal{W}\left[\mathbf{n}_{0}\right]$, one gets

$$
\mathbf{T}\left[\mathbf{n}_{0}\right]=\frac{1}{2}\left[\begin{array}{cc}
-v \mathcal{K}^{2} & -\varepsilon^{-1} \mathcal{K} \\
\mu^{-1} \mathcal{K} & -v \mathcal{K}^{2}
\end{array}\right]+\frac{1}{2}\left[\begin{array}{cc}
v \mathcal{K}^{2} & -\varepsilon^{-1} \mathcal{K} \\
\mu^{-1} \mathcal{K} & v \mathcal{K}^{2}
\end{array}\right] .
$$

The surface integral on the left-hand section of (7) can be computed if flux $\mathbf{T}\left[\mathbf{n}_{0}\right] \mathbf{F}$ is already known on all faces of boundary $\partial \Omega_{i}$. For this aim, two subvectors of $\mathbf{F}$ are considered; one on the internal and the other on the external side of each face. Both quantities are acquired by an interpolation procedure centered on the respective face side. Plugging (8)-(10) into (7), we derive

$$
\partial_{t} \mathbf{F}+\mathbf{Z F}=\mathbf{Y}
$$

for $\mathbf{Z}$ a bloc-diagonal matrix with all media properties and $\mathbf{Y}$ a term with the source contributions of every cell. Note that $\mathbf{Z}$ is inverted only once at the beginning of the simulation. To complete the proposed FVTD method, time derivatives in (11) are precisely evaluated by the leapfrog predictor-corrector scheme

$$
\mathbf{F}^{n+1 / 2}=\mathbf{F}^{n}-0.5 \Delta t \mathbf{A} \mathbf{F}^{n} \text { and } \mathbf{F}^{n+1}=\mathbf{F}^{n}-0.5 \Delta t \mathbf{A} \mathbf{F}^{n+1 / 2} \text {, }
$$

in which $\mathbf{A}$ is the conventional update matrix for vector $\mathbf{F}$.

\section{Domain DeComposition AND STABILITY ANAlysis}

A significant aspect in the analysis of structures with symmetries/periodicities is the separation of the basic domain into smaller regions, where a numerical scheme can be more easily and economically applied [13]. Herein, to avoid unnecessary discretizations of identical areas, a 3-D domain decomposition algorithm is developed. Our space is divided into $K$ non-overlapping subdomains. Specifically, for axially symmetric problems, these subdomains occupy the intermediate region between successive cylindrical surfaces toward the radial direction, while for planar symmetries/periodicities, they include one unit cell. Hence, when two subdomains $(i=1,2)$ are considered, at their interface $\mathbf{E}_{1}$ and $\mathbf{H}_{2}$ (dually $\mathbf{E}_{2}$ and $\mathbf{H}_{1}$ ) vectors are related by

$$
\begin{gathered}
\hat{\mathbf{n}}_{1} \times \mu_{r, 1}^{-1} \nabla \times \mathbf{E}_{1}=-\mu_{0} \hat{\mathbf{n}}_{2} \times \partial_{t} \mathbf{H}_{2}, \\
\hat{\mathbf{n}}_{2} \times \varepsilon_{r, 2}^{-1} \nabla \times \mathbf{H}_{2}=\varepsilon_{0} \hat{\mathbf{n}}_{1} \times \partial_{t} \mathbf{E}_{1} .
\end{gathered}
$$

Moreover, for the artificial boundary oscillations, an equivalent surface current is employed, i.e.

$$
\mathbf{J}_{s, i}=\hat{\mathbf{n}}_{i} \times \mu_{r, 1}^{-1} \nabla \times \mathbf{E}_{i} \quad \text { for } \quad i=1,2,
$$




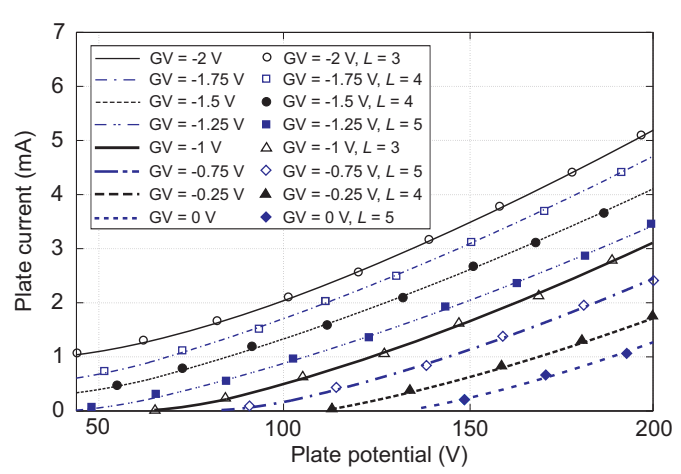

Fig. 3. Simulated plate current curves versus plate potential of the triode for three different values of grid voltages (continuous and dashed lines: VFEM; circles and squares: proposed FVTD method for several $L$ orders).

and $\mathbf{n}_{i}$ outward normal unit vectors. The proposed methodology together with (13)-(15) is proven stable for all types of numerical simulations. Actually, von Neumann's analysis shows that

$$
v \Delta t \leq(2 L+1) \sin ^{-1}(0.65)\left(\sum_{l=u}^{w} \sum_{m=u}^{w} \frac{g(u, v, w)}{\Delta l \Delta m}\right)^{-1 / 2}
$$

with the summations denoting a consecutive cyclic permutation of $(u, v, w)$. Notice the less strict character of (16) than the FDTD Courant condition, due to the dependence on order $L$. This implies that time-steps can be chosen quite larger, without arising any oscillatory exponential modes. Alternatively, stability can be certified via the energy inequality method [14], which gives

$$
\left\|\mathbf{F}^{n+1}(t)\right\| \leq\left\|\mathbf{F}^{n}(t)\right\|+\sum_{\tau=0}^{n} a\left\|\mathbf{F}^{\tau+1}\right\|,
$$

where $a$ is a growth factor. Inequality (17) guarantees that $\mathbf{F}(t)$ does not augment above a certain limit, namely the system's energy is conserved. Also, the dispersion relation of the enhanced method is greatly improved owing to the parameterized adjustment of its spectral resolution and the bloc-diagonal matrix $\mathbf{Z}$, as compared to the usual $G_{\text {FDTD }}$ one. So, we conclude to

$$
\sin ^{2}\left(\frac{\omega \Delta t}{2}\right)=\frac{21(\Delta t)^{3 / 5}}{8^{L+2} v} G_{\text {FDTD }},
$$

which, as clearly observed from Fig. 2, is found to overwhelm both second- and higher-order FDTD schemes.

\section{NUMERICAL Results}

The proposed technique is validated via different real-world EMC structures. Focusing on reconfigurability, we exploit the merits of $L$ th-order operator (2) to select optimal stencils near acute angles or geometric oddities. The first application is the typical triode of Fig. 1a. Its dimensions are: $a=5.6 \mathrm{~cm}, b=2$ $\mathrm{cm}, c=1.8 \mathrm{~cm}, d=1 \mathrm{~cm}, e=0.8 \mathrm{~cm}, g=0.6 \mathrm{~cm}, h=0.2 \mathrm{~cm}$, and $m=4.4 \mathrm{~cm}$, while for open-boundary termination a 6-cell thick PML is employed. In our simulations, $L=3,4$ or 5 which lead to the very coarse $42 \times 26 \times 40$ lattice. Figure 3 gives the triode plate current versus plate potential for various triode grid voltages (GV). As detected, our algorithm is very accurate compared to the reference results of a second-order vector finite element method (VFEM), which however needs a mesh of $8.25 \times 10^{5}$ degrees of freedom. In fact, the overhead of the new method is around $700 \mathrm{MB}$ of RAM, while the VFEM one is $65 \%$ larger, i.e. $1.2 \mathrm{~GB}$ of RAM. Moreover, due to its explicit profile the enhanced FVTD method is almost 5.2 faster than the

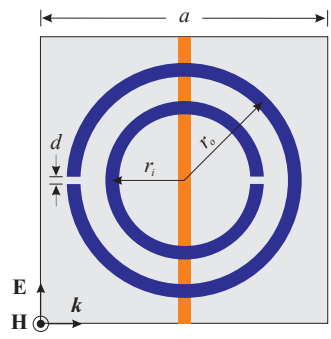

(a)

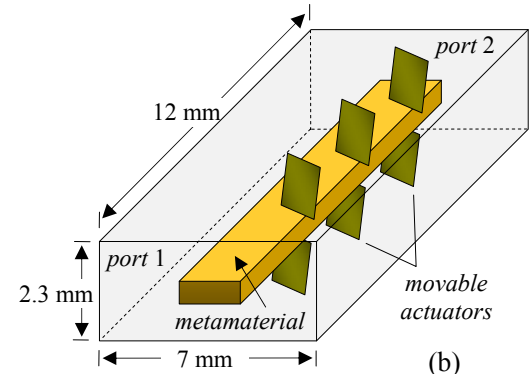

(b)
Fig. 4. (a) Top view of a composite circular SRR-wire metamaterial unit cell and (b) a reconfigurable 2-port coaxial waveguide with movable actuators and a periodically-loaded metamaterial slab.
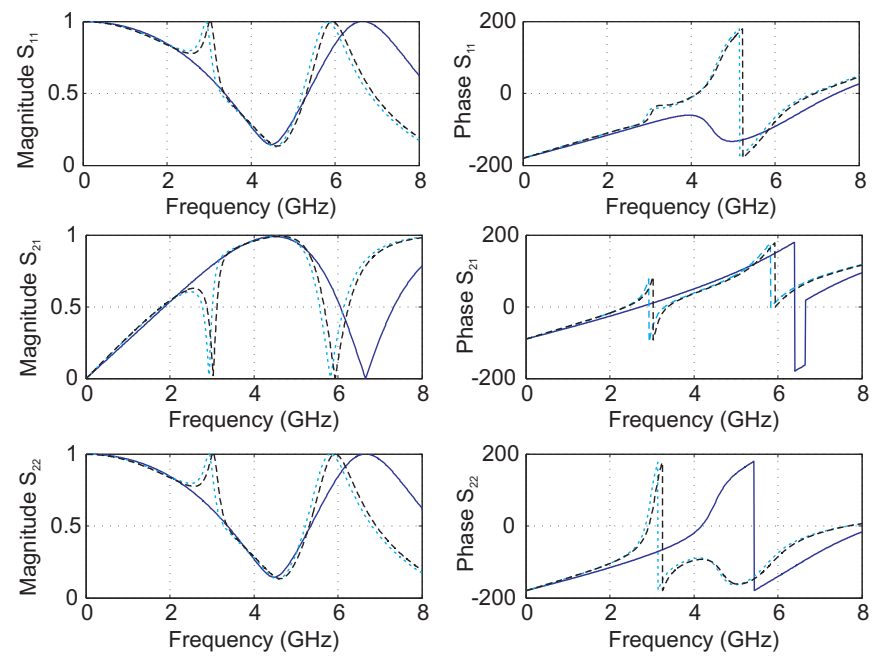

Fig. 5. Normalized magnitude and phase (in degrees) of several $S$-parameters for a $10 \times 10$ metamaterial based on the unit cell of Fig. 4 a (blue straight line: FDTD method with $342 \times 342 \times 36$ cells; cyan dotted line: VFEM with $6 \times 10^{5}$ degrees of freedom; black dashed line: proposed FVTD method with $48 \times 48 \times 10$ cells).

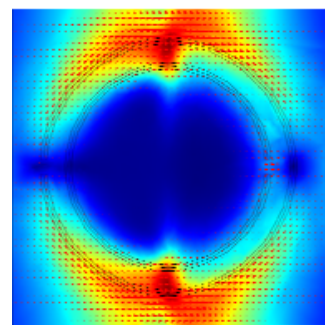

(a)

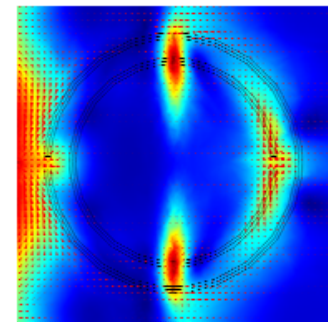

(b)
Fig. 6. Snapshots of the Poynting vector at (a) $4.5 \mathrm{GHz}$ and (b) $5.93 \mathrm{GHz}$.

other setups on a $3.2 \mathrm{GHz}$ dual-processor Intel ${ }^{\mathrm{TM}}$ Xeon system.

The second example examines a $10 \times 10$ periodical metamaterial, whose unit cell is shown in Fig. 4a. The dimensions of the 3-D cell, comprising a circular split-ring resonator (SRR) above a thin wire at a distance of $1.875 \mathrm{~mm}$, are: $a=15 \mathrm{~mm}, r_{o}$ $=6 \mathrm{~mm}, r_{i}=4.8 \mathrm{~mm}, d=0.1 \mathrm{~mm}$, and a circular cross-section of $0.02 \mathrm{~mm}$. Figure 5 compares the magnitude and phase of three $S$-parameters, as computed via the FDTD technique, the VFEM, and the new formulation $(L=4)$ with a much coarser grid. Note the lack of the FDTD approach to follow the promising agreement of the other two schemes, especially at high frequencies. Moreover, Fig. 6 proves that our method can produce very smooth Poynting vector snapshots, both at the full propagation (Fig. 6a) or the nearly no propagation (Fig. 6b) case. 

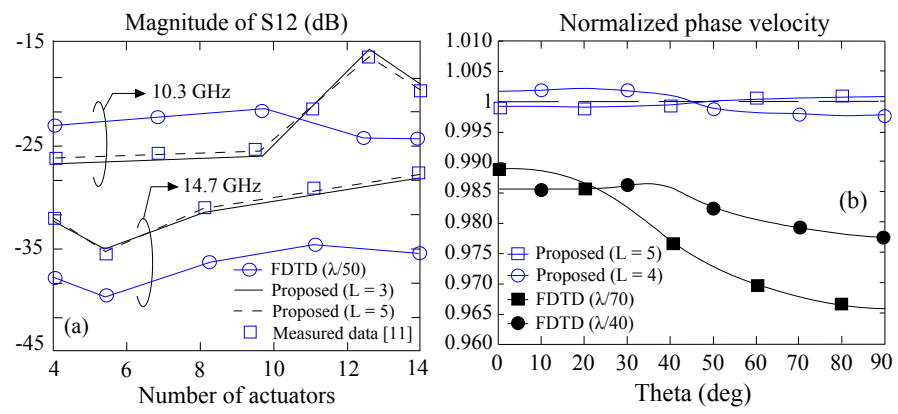

Fig. 7. (a) Magnitude of $S_{12}$-parameter versus the number of actuators in the reconfigurable 2-port waveguide and (b) normalized phase velocity.

TABLE I

First RESONANCE OF THE WAVEGUIDE FOR THREE SETS OF ACTUATORS

\begin{tabular}{cccccc}
\hline $\begin{array}{c}\text { Ref. [12] } \\
(\mathrm{GHz})\end{array}$ & Method & $\begin{array}{c}\text { Comp. } \\
(\mathrm{GHz})\end{array}$ & $\begin{array}{c}\text { Error } \\
(\%)\end{array}$ & $\begin{array}{c}\text { CPU } \\
\text { Time }\end{array}$ & $\begin{array}{c}\text { Maximum } \\
\text { Dispersion }\end{array}$ \\
\hline 4 Actuators & FDTD & 10.873 & 7.06 & $10.2 \mathrm{~h}$ & $2.05892 \times 10^{-1}$ \\
10.156 & Proposed & 10.155 & 0.01 & $1.4 \mathrm{~h}$ & $1.98591 \times 10^{-11}$ \\
\hline 6 Actuators & FDTD & 11.249 & 9.34 & $13.8 \mathrm{~h}$ & 3.89792 \\
12.408 & Proposed & 12.411 & 0.03 & $1.7 \mathrm{~h}$ & $2.67362 \times 10^{-10}$ \\
\hline 10 Actuators & FDTD & 17.774 & 12.83 & $15.6 \mathrm{~h}$ & 7.11032 \\
15.753 & Proposed & 15.742 & 0.07 & $2.2 \mathrm{~h}$ & $5.20265 \times 10^{-10}$ \\
\hline
\end{tabular}

Operation as a microstrip
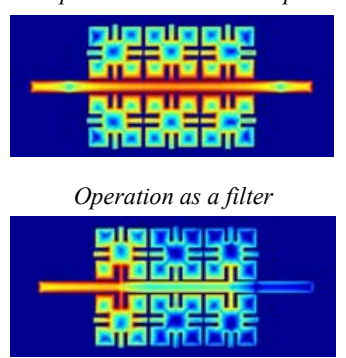

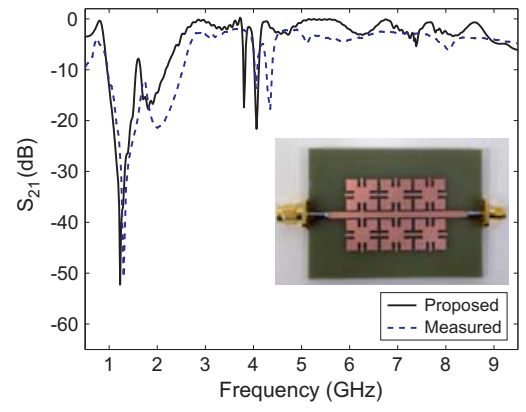

Fig. 8. Snapshots of surface current distribution and magnitude of $S_{21}$ simulated and measured results for a uniform cross/gap EBG-loaded microwave filter.

Next, we proceed to the reconfigurable 2-port waveguide of Fig. 4b, which involves a movable set of actuators (for selective mode propagation), placed on a slab composed of the previous metamaterial. The variation of the $S_{12}$-parameter and the first resonance of the structure regarding the number of actuators, are presented in Fig. 7a and Table I. Evidently, the proposed technique is considerably more accurate and cost-effective in overall CPU time overhead, attaining also notably low dispersion errors and consistent phase velocities, as observed in Fig. $7 \mathrm{~b}$.

Finally, our analysis studies the uniform and non-uniform cross/gap EBG microwave filters of Fig. 1b. The main element of these devices is a $2.8 \mathrm{~mm}$-wide microstrip line loaded with two cross EBG unit cells which render the structure reconfigurable in terms of its frequency bands. The height of the unit cell is $15.24 \mathrm{~mm}$, the thickness of the $\varepsilon_{r}=4.5$ substrate is $1.5 \mathrm{~mm}$, the filter's length is $13.716 \mathrm{~cm}$ and its width $6.456 \mathrm{~cm}$. For the uniform structure, $L=3$ and the lattice has $68 \times 44 \times 16$ cells, unlike the $615 \times 354 \times 126$ FDTD grid. The results of Fig. 8 , compared to measurements from our fabricated prototype, indicate the accuracy of our technique. In contrast, the non-uniform case is designed via a Chebyshev window. The length of the device is divided into equal intervals and after computing the value of the Chebyshev function, only the $n$ higher terms ( $n$ is the EBG unit cell number on one side of the filter) is selected. The $S_{21}$ -

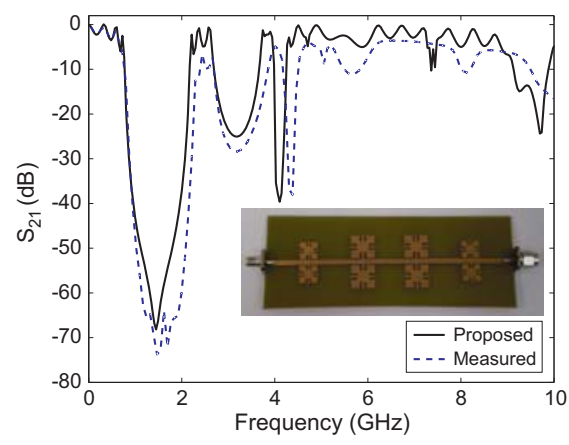

Fig. 9. Magnitude of the $S_{21}$-parameter for a non-uniform cross EBG-loaded ( $n$ =4) microwave filter.

parameter of this complex device, shown in Fig. 9, certifies, again, the merits of the advanced FVTD algorithm for $L=4$.

\section{CONCLUSIONS}

A 3-D FVTD method with a controllable spectral accuracy for the reliable study of complicated amplification and reconfigurable devices has been presented in this paper. The new technique introduces a set of general spatial approximators, so leading to optimal stencils. For its elaborate validation, the algorithm has been successfully applied to several realistic setups.

\section{REFERENCES}

[1] N. T. Tung, V. D. Lam, M. H. Cho, J. W. Park, S. J. Lee, W. H. Jang, and Y. P. Lee, "Effects of the electric component on combined metamaterial structure," IEEE Trans. Magn., vol. 45, pp. 4310-4313, Oct. 2009.

[2] V. De Santis, M. Feliziani, and F. Maradei, "Safety assessment of UWB radio systems for body area network by the FD ${ }^{2}$ TD method," IEEE Trans. Magn., vol. 46, no. 8, pp. 3245-3248, Aug. 2010.

[3] Y. Li, J. Zhu, Q. Yang, Z. Wei, Y. Guo, and Y. Wang, "Measurement of soft magnetic composite material using an improved 3-D tester with novel sensing coils," IEEE Trans. Magn., vol. 46, no. 6, pp. 1971-1974, 2010.

[4] J. Lu, B. Zhu, and D. Thiel, "Full wave solution for Intel CPU in EMC investigations," IEEE Trans. Magn., vol. 46, no. 8, pp. 3405-3408, 2010.

[5] T. Ohtani, K. Taguchi, T. Kashiwa, Y. Kanai, and J. Cole, "Scattering analysis of large-scale coated cavity using the nonstandard FDTD method," IEEE Trans. Magn., vol. 45, no. 3, pp. 1296-1299, Mar. 2009.

[6] L. Bernard, R. Torrado, and L. Pichon, "Efficient implementation of the UPML in the generalized finite-difference time-domain method," IEEE Trans. Magn., vol. 46, no. 8, pp. 3492-3495, Aug. 2010.

[7] L. Prokopeva, J. Borneman, and A. Kildishev, "Optical dispersion models for time-domain modeling of meta-dielectric nanostructures," IEEE Trans. Magn., vol. 47, no. 5, pp. 1150-1153, May 2011.

[8] J. Scott, S. Roxenborg, and A. Parker, "An improved triode model," in Proc. 6th Regional Convention of the Audio Engineering Society, pp. 19, 1996, Melbourne, Australia.

[9] R. Holland, V. Cable, and L. Wilson, "Finite-volume time-domain (FVTD) techniques for EM scattering," IEEE Trans. Electromagn. Compat., vol. 33, no. 4, pp. 281-294, Nov. 1991.

[10] K. Yee and J. Chen, "The finite-difference time-domain and the finitevolume time-domain methods in solving Maxwell's equations," IEEE Trans. Antennas Propag., vol. 45, no. 3, pp. 354-363, Mar. 1997.

[11] D. Baumann, C. Fumeuax, and R. Vahldieck, "Field-based scattering matrix extraction scheme for the FVTD method exploiting a fluxsplitting algorithm," IEEE Trans. Microw. Theory Tech., vol. 53, no. 11, pp. 3595-3605, Nov. 2005.

[12] G. Bozza, D. Caviglia, L. Ghelardoni, and M. Pastorino, "Cell-centered finite-volume time-domain method," IEEE Microw. Wireless Compon. Lett., vol. 20, no. 9, pp. 477-479, Sep. 2010.

[13] D. Firsov, J. LoVetri, O. Jeffrey, V. Okhmatovski, C. Gilmore, and W. Chamma, "High-order FVTD schemes for Maxwell's equations," ACES J., vol. 22, pp. 71-82, 2007.

[14] C. Bommaraju, W. Ackermann, and T. Weiland, "Convergence of error in FVTD methods on tetrahedral meshed in 3D," in Proc. Applied Electromagn. Conf. $(A E M C)$, pp. 1-4, 2009. 